TRANSACTIONS OF THE

AMERICAN MATHEMATICAL SOCIETY

Volume 364, Number 10, October 2012, Pages 5369-5394

S 0002-9947(2012)05514-0

Article electronically published on April 30, 2012

\title{
ON THE HAUSDORFF DIMENSION OF THE ESCAPING SET OF CERTAIN MEROMORPHIC FUNCTIONS
}

\author{
WALTER BERGWEILER AND JANINA KOTUS
}

\begin{abstract}
Let $f$ be a transcendental meromorphic function of finite order $\rho$ for which the set of finite singularities of $f^{-1}$ is bounded. Suppose that $\infty$ is not an asymptotic value and that there exists $M \in \mathbb{N}$ such that the multiplicity of all poles, except possibly finitely many, is at most $M$. For $R>0$ let $I_{R}(f)$ be the set of all $z \in \mathbb{C}$ for which $\liminf _{n \rightarrow \infty}\left|f^{n}(z)\right| \geq R$ as $n \rightarrow \infty$. Here $f^{n}$ denotes the $n$-th iterate of $f$. Let $I(f)$ be the set of all $z \in \mathbb{C}$ such that $\left|f^{n}(z)\right| \rightarrow \infty$ as $n \rightarrow \infty$; that is, $I(f)=\bigcap_{R>0} I_{R}(f)$. Denote the Hausdorff dimension of a set $A \subset \mathbb{C}$ by $\operatorname{HD}(A)$. It is shown that $\lim _{R \rightarrow \infty} \operatorname{HD}\left(I_{R}(f)\right) \leq$ $2 M \rho /(2+M \rho)$. In particular, $\operatorname{HD}(I(f)) \leq 2 M \rho /(2+M \rho)$. These estimates are best possible: for given $\rho$ and $M$ we construct a function $f$ such that $\operatorname{HD}(I(f))=2 M \rho /(2+M \rho)$ and $\operatorname{HD}\left(I_{R}(f)\right)>2 M \rho /(2+M \rho)$ for all $R>0$.

If $f$ is as above but of infinite order, then the area of $I_{R}(f)$ is zero. This result does not hold without a restriction on the multiplicity of the poles.
\end{abstract}

\section{INTRODUCTION AND MAIN RESULTS}

The Fatou set $F(f)$ of a (non-linear) function $f$ meromorphic in the plane is defined as the set of all points $z \in \mathbb{C}$ such that the iterates $f^{k}$ of $f$ are defined and form a normal family in some neighbourhood of $z$. Furthermore, $J(f)=\widehat{\mathbb{C}} \backslash F(f)$, where $\widehat{\mathbb{C}}=\mathbb{C} \cup\{\infty\}$ is called the Julia set of $f$ and

$$
I(f)=\left\{z \in \mathbb{C}: f^{n}(z) \rightarrow \infty \text { as } n \rightarrow \infty\right\}
$$

is called the escaping set of $f$. In addition to these sets, we shall also consider for $R>0$ the set

$$
I_{R}(f)=\left\{z \in \mathbb{C}: \liminf _{n \rightarrow \infty}\left|f^{n}(z)\right| \geq R\right\} .
$$

Note that

$$
I(f)=\bigcap_{R>0} I_{R}(f) .
$$

It was shown by Eremenko [7] for entire $f$ and by Domínguez [6] for transcendental meromorphic $f$ that $I(f) \neq \emptyset$ and that $J(f)=\partial I(f)$. For an introduction to the iteration theory of transcendental meromorphic functions we refer to [4]. Results on the Hausdorff dimension of Julia sets and related sets are surveyed in 14, 25.

Received by the editors January 21, 2009 and, in revised form, May 13, 2010 and November $22,2010$.

2010 Mathematics Subject Classification. Primary 37F10; Secondary 30D05, 30D15.

The authors were supported by the EU Research Training Network CODY. The first author was also supported by the G.I.F., the German-Israeli Foundation for Scientific Research and Development, Grant G-809-234.6/2003, the ESF Research Networking Programme HCAA and the Deutsche Forschungsgemeinschaft, Be 1508/7-1. The second author was also supported by PW Grant 504G 11200011000 and Polish MNiSW Grant "Chaos, fraktale i dynamika konforemna II". 
The set of singularities of the inverse function $f^{-1}$ of $f$ coincides with the set of critical values and asymptotic values of $f$. We denote the set of finite singularities of $f^{-1}$ by $\operatorname{sing}\left(f^{-1}\right)$. The Eremenko-Lyubich class $\mathcal{B}$ consists of all meromorphic functions for which $\operatorname{sing}\left(f^{-1}\right)$ is bounded. Eremenko and Lyubich [9, Theorem 1] proved that if $f \in \mathcal{B}$ is entire, then $I(f) \subset J(f)$. This result was extended to meromorphic functions in $\mathcal{B}$ by Rippon and Stallard 21. Actually the proof yields that $I_{R}(f) \subset J(f)$ if $f \in \mathcal{B}$ and $R$ is sufficiently large.

For a subset $A$ of $\mathbb{C}$ we denote by $\operatorname{HD}(A)$ the Hausdorff dimension of $A$ and by area $(A)$ the two-dimensional Lebesgue measure of $A$. McMullen [18] proved that $\operatorname{HD}\left(J\left(\lambda e^{z}\right)\right)=2$ for $\lambda \in \mathbb{C} \backslash\{0\}$ and that area $(J(\sin (\alpha z+\beta z)))>0$ for $\alpha, \beta \in \mathbb{C}$, $\alpha \neq 0$. His proof shows that the conclusion holds with $J(\cdot)$ replaced by $I(\cdot)$. Note that the functions considered by McMullen are in the class $\mathcal{B}$ so that the escaping set is contained in the Julia set.

The order $\rho(f)$ of a meromorphic function $f$ is defined by

$$
\rho(f)=\limsup _{r \rightarrow \infty} \frac{\log T(r, f)}{\log r},
$$

where $T(r, f)$ denotes the Nevanlinna characteristic of $f$; see [10, 11, 19] for the notation of Nevanlinna theory. If $f$ is entire, then we may replace $T(r, f)$ by $\log M(r, f)$ here, where $M(r, f)=\max _{z=r}|f(z)|$. Thus for entire $f$ we have [11, p. 18]

$$
\rho(f)=\limsup _{r \rightarrow \infty} \frac{\log \log M(r, f)}{\log r} .
$$

It is easy to see that $\rho\left(\lambda e^{z}\right)=\rho(\sin (\alpha z+\beta z))=1$ for $\lambda, \alpha, \beta \in \mathbb{C}, \lambda, \alpha \neq 0$.

McMullen's result that $\operatorname{HD}\left(J\left(\lambda e^{z}\right)\right)=2$ was substantially generalized by Barański [2] and Schubert [22] who proved that if $f \in \mathcal{B}$ is entire and $\rho(f)<\infty$, then $\operatorname{HD}(J(f))=2$. They actually showed that $\operatorname{HD}\left(I_{R}(f)\right)=2$ for all $R>0$ under these hypotheses. Their proofs, which make use of a logarithmic change of variable introduced to complex dynamics by Eremenko and Lyubich, show that the conclusion holds more generally for meromorphic functions in $\mathcal{B}$ which have finite order and for which $\infty$ is an asymptotic value. In fact, such functions have a logarithmic singularity over $\infty$ and their dynamics are in many ways similar to those of entire functions; see, e.g., $[\underline{3}$ or $[5]$.

The purpose of this paper is to show that the situation is very different for meromorphic functions of class $\mathcal{B}$ for which $\infty$ is not an asymptotic value.

Theorem 1.1. Let $f \in \mathcal{B}$ be a transcendental meromorphic function satisfying $\rho=\rho(f)<\infty$. Suppose that $\infty$ is not an asymptotic value and that there exists $M \in \mathbb{N}$ such that the multiplicity of all poles, except possibly finitely many, is at most $M$. Then

$$
\operatorname{HD}(I(f)) \leq \frac{2 M \rho}{2+M \rho}
$$

and

$$
\lim _{R \rightarrow \infty} \operatorname{HD}\left(I_{R}(f)\right) \leq \frac{2 M \rho}{2+M \rho} .
$$

Note that the right hand side of (1.1) and (1.2) is strictly less than 2. Thus, as explained above, the conclusion does not hold if $\infty$ is an asymptotic value. 
Clearly $I_{S}(f) \subset I_{R}(f)$ if $S>R$. Hence $\operatorname{HD}\left(I_{R}(f)\right)$ is a non-increasing function of $R$ and thus the limit in (1.2) exists. Clearly (1.1) follows from (1.2) so that it suffices to prove (1.2).

If $\rho=0$, then (1.1) says that $\operatorname{HD}(I(f))=0$. This contrasts with a result of Stallard [24] who proved that $\operatorname{HD}(J(f))>0$ for every transcendental meromorphic function $f$. Examples of meromorphic functions of order 0 which satisfy the hypotheses of Theorem 1.1 exist. In fact, there are such examples not only in the Eremenko-Lyubich class $\mathcal{B}$, but also in the smaller Speiser class $\mathcal{S}$ of meromorphic functions for which $\operatorname{sing}\left(f^{-1}\right)$ is finite; see [1, Section 5], 8] and [15. Langley [15, Example 2.1] gave an example where the multiplicities of the poles are bounded and $\operatorname{sing}\left(f^{-1}\right)$ consists of only two points. Composing this with a Möbius transformation we obtain an example where all poles are simple and $\operatorname{sing}\left(f^{-1}\right)$ consists of three points.

We note that elliptic functions are in $\mathcal{B}$ and have order 2. It was shown in 13 , Theorem 1.2] that if $M$ denotes the maximal multiplicity of the poles of an elliptic function $f$, then $\operatorname{HD}(I(f)) \leq 2 M /(1+M)$. Inequality (1.1) generalizes this result. On the other hand, it was shown in [12, Example 3] that if $f$ is an elliptic function such that the closure of the postcritical set is disjoint from the set of poles, then $\operatorname{HD}(J(f)) \geq 2 M /(1+M)$. The argument actually shows that $\mathrm{HD}(I(f)) \geq 2 M /(1+M)$. Thus (1.1) is best possible if $\rho=2$. The following result shows that Theorem 1.1 is best possible for all values of $\rho$.

Theorem 1.2. Let $0<\rho<\infty$ and $M \in \mathbb{N}$. Then there exists a meromorphic function $f \in \mathcal{B}$ of order $\rho$ for which all poles have multiplicity $M$ and for which $\infty$ is not an asymptotic value such that

$$
\mathrm{HD}(I(f))=\frac{2 M \rho}{2+M \rho}
$$

and

$$
\operatorname{HD}\left(I_{R}(f)\right)>\frac{2 M \rho}{2+M \rho}
$$

for all $R>0$.

Note that the right hand side of (1.3) and (1.4) tends to 2 if $\rho \rightarrow \infty$ or if $\rho>0$ and $M \rightarrow \infty$. Therefore we cannot expect the Hausdorff dimension of $I(f)$ or $I_{R}(f)$ to be strictly less than 2 if $\rho=\infty$ or if there are poles of arbitrarily high multiplicity.

In fact, a modification of the proof of Theorem 1.2 shows that there exists a meromorphic function $f \in \mathcal{B}$ of infinite order which has only simple poles and for which $\infty$ is not an asymptotic value such that $\operatorname{HD}(I(f))=2$; see the remark at the end of section 6 .

However, we have the following result.

Theorem 1.3. Let $f \in \mathcal{B}$ be a transcendental meromorphic function for which $\infty$ is not an asymptotic value. Suppose that there exists $M \in \mathbb{N}$ such that all poles of $f$ have multiplicity at most $M$. Then

$$
\text { area }\left(I_{R}(f)\right)=0
$$

for sufficiently large $R$. In particular,

$$
\text { area }(I(f))=0
$$


The proof of Theorem 1.3 uses well-known techniques; see 23. for a similar argument. In fact, as kindly pointed out to us by Lasse Rempe, Theorem [1.3 is implicitly contained in [20, Theorem 7.2]. However, we shall include the short proof of Theorem 1.3 for completeness.

Finally we show that the hypothesis on the multiplicity of the poles is essential in Theorem 1.3 .

Theorem 1.4. There exists a transcendental meromorphic $f \in \mathcal{B}$ for which $\infty$ is not an asymptotic value and for which

$$
\operatorname{area}(I(f))>0 \text {. }
$$

\section{NOTATION AND PRELIMINARY LEMMAS}

The diameter of a set $K \subset \mathbb{C}$ is denoted by $\operatorname{diam}(K)$. Later we will also use the area and diameter with respect to the spherical metric $\chi$. We will denote them by $\operatorname{area}_{\chi}(K)$ and $\operatorname{diam}_{\chi}(K)$, respectively.

For $a \in \mathbb{C}$ and $r, R>0$ we use the notation $D(a, r)=\{z \in \mathbb{C}:|z-a|<r\}$ and $B(R)=\{z \in \mathbb{C}:|z|>R\} \cup\{\infty\}$.

The following lemma is known as Koebe's distortion theorem and Koebe's onequarter theorem.

Lemma 2.1. Let $g: D(a, r) \rightarrow \mathbb{C}$ be univalent, $0<\lambda<1$ and $z \in D(a, \lambda r)$. Then

$$
\begin{gathered}
|g(z)-g(a)| \leq \frac{\lambda}{(1-\lambda)^{2}}\left|g^{\prime}(a)\right| r \\
\frac{1-\lambda}{(1+\lambda)^{3}}\left|g^{\prime}(a)\right| \leq\left|g^{\prime}(z)\right| \leq \frac{1+\lambda}{(1-\lambda)^{3}}\left|g^{\prime}(a)\right|
\end{gathered}
$$

and

$$
g(D(a, r)) \supset D\left(g(a), \frac{1}{4}\left|g^{\prime}(a)\right| r\right)
$$

Koebe's theorem is usually only stated for the special case that $a=0, r=1$, $g(0)=0$ and $g^{\prime}(0)=1$, but the above version follows immediately from this special case.

The following result is due to Rippon and Stallard [21, Lemma 2.1].

Lemma 2.2. Let $f \in \mathcal{B}$ be transcendental. If $R>0$ such that $\operatorname{sing}\left(f^{-1}\right) \subset D(0, R)$, then all components of $f^{-1}(B(R))$ are simply connected. Moreover, if $\infty$ is not an asymptotic value of $f$, then all components of $f^{-1}(B(R))$ are bounded and contain exactly one pole of $f$.

The following result is known as Iversen's theorem ([10, p. 171] or [19, p. 292]).

Lemma 2.3. Let $f$ be a transcendental meromorphic function for which $\infty$ is not an asymptotic value. Then $f$ has infinitely many poles.

Let $\left(a_{j}\right)$ be a sequence of non-zero complex numbers satisfying

$$
\lim _{j \rightarrow \infty}\left|a_{j}\right|=\infty .
$$

Then

$$
\sigma=\sigma\left(\left(a_{j}\right)\right)=\inf \left\{\mu>0: \sum_{j=1}^{\infty} \frac{1}{\left|a_{j}\right|^{\mu}}<\infty\right\}
$$


is called the exponent of convergence of the sequence $\left(a_{j}\right)$. Here we put $\inf \emptyset=\infty$, meaning that $\sigma=\infty$ if $\sum_{j=1}^{\infty}\left|a_{j}\right|^{-\mu}=\infty$ for all $\mu>0$. Denote by $n(r)$ the number of $a_{j}$ in $\overline{D(0, r)}$ and put

$$
N(r)=\int_{0}^{r} \frac{n(t)}{t} d t .
$$

Then [10, Chapter 2, Theorems 1.1 and 1.8] for $\mu>0$ the series and the two integrals

$$
\sum_{j=1}^{\infty} \frac{1}{\left|a_{j}\right|^{\mu}}, \quad \int_{1}^{\infty} \frac{n(t)}{t^{\mu+1}} d t \quad \text { and } \quad \int_{1}^{\infty} \frac{N(t)}{t^{\mu+1}} d t
$$

are all convergent or all divergent and

$$
\sigma=\limsup _{r \rightarrow \infty} \frac{\log n(r)}{\log r}=\limsup _{r \rightarrow \infty} \frac{\log N(r)}{\log r} .
$$

Recall that the Nevanlinna characteristic $T(r, f)$ is defined by

$$
T(r, f)=N(r, f)+m(r, f),
$$

where

$$
m(r, f)=\int_{0}^{2 \pi} \log ^{+}\left|f\left(r e^{i \vartheta}\right)\right| d \vartheta
$$

is the Nevanlinna proximity function and $N(r, f)$ is the counting function formed with the sequence $\left(a_{j}\right)$ of poles of $f$ as above. (If 0 is a pole, there is a slight modification.) The following well-known lemma follows immediately from (2.4) and the definition of the order.

Lemma 2.4. Let $f$ be a transcendental meromorphic function and let $\sigma$ be the exponent of convergence of the non-zero poles of $f$. Then $\sigma \leq \rho(f)$.

We mention that a result of Teichmüller [26] says that if $f \in \mathcal{B}$ is transcendental, if $\infty$ is not an asymptotic value of $f$ and if there exists $M \in \mathbb{N}$ such that all poles of $f$ have multiplicity at most $M$, then $m(r, f)=O(1)$. Thus we have $T(r, f)=N(r, f)+O(1)$ as $r \rightarrow \infty$. It follows from Teichmüller's result and (2.4) that if $f$ is as in Theorem 1.1, then the exponent of convergence of the non-zero poles of $f$ is actually equal to $\rho(f)$.

\section{Proof of Theorem 1.1}

By Lemma 2.3. $f$ has infinitely many poles. Let $\left(a_{j}\right)$ be the sequence of poles of $f$, ordered such that $\left|a_{j}\right| \leq\left|a_{j+1}\right|$ for all $j$, and let $m_{j}$ be the multiplicity of $a_{j}$. Then

$$
f(z) \sim\left(\frac{b_{j}}{z-a_{j}}\right)^{m_{j}} \quad \text { as } \quad z \rightarrow a_{j}
$$

for some $b_{j} \in \mathbb{C} \backslash\{0\}$. We may assume that $\left|a_{j}\right| \geq 1$ for all $j \in \mathbb{N}$. Let $R_{0}>1$ such that $\operatorname{sing}\left(f^{-1}\right) \subset D\left(0, R_{0}\right)$ and $|f(0)|<R_{0}$.

Lemma 2.2 says that if $R \geq R_{0}$, then all the components of $f^{-1}(B(R))$ are bounded and simply connected and each component contains exactly one pole. We denote the component containing $a_{j}$ by $U_{j}$ and choose a conformal map

$$
\phi_{j}: U_{j} \rightarrow D\left(0, R^{-1 / m_{j}}\right)
$$

satisfying $\phi_{j}\left(a_{j}\right)=0$. Then $\left|f(z) \phi_{j}(z)^{m_{j}}\right| \rightarrow 1$ as $z$ approaches the boundary of $U_{j}$. Since $\left|f(z) \phi_{j}(z)^{m_{j}}\right|$ remains bounded near $a_{j}$ and is non-zero in $U_{j}$, we can 
deduce from the maximum principle that $\left|f(z) \phi_{j}(z)^{m_{j}}\right|=1$ for all $z \in U_{j} \backslash\left\{a_{j}\right\}$ and that $\left|\phi_{j}^{\prime}\left(a_{j}\right)\right|=1 /\left|b_{j}\right|$. We may actually normalize $\phi_{j}$ such that $\phi_{j}^{\prime}\left(a_{j}\right)=1 / b_{j}$. Denote the inverse function of $\phi_{j}$ by $\psi_{j}$. Since $\psi_{j}(0)=a_{j}$ and $\psi_{j}^{\prime}(0)=b_{j}$ we deduce from (2.3) that

$$
U_{j}=\psi_{j}\left(D\left(0, R^{-1 / m_{j}}\right)\right) \supset D\left(a_{j}, \frac{1}{4}\left|b_{j}\right| R^{-1 / m_{j}}\right) \supset D\left(a_{j}, \frac{1}{4 R}\left|b_{j}\right|\right) .
$$

Since $|f(0)|<R$ we have $0 \notin U_{j}$. Thus (3.1) implies in particular that

for all $R \geq R_{0}$ and hence that

$$
\frac{1}{4 R}\left|b_{j}\right| \leq\left|a_{j}\right|
$$

$$
\left|b_{j}\right| \leq 4 R_{0}\left|a_{j}\right| .
$$

We note that $\psi_{j}$ actually extends to a map univalent in $D\left(0, R_{0}^{-1 / m_{j}}\right)$. Applying (2.1) with

we find that

$$
\lambda=\left(\frac{R}{R_{0}}\right)^{-1 / m_{j}}=\left(\frac{R_{0}}{R}\right)^{1 / m_{j}}
$$

$$
U_{j} \subset D\left(a_{j}, \frac{\lambda}{(1-\lambda)^{2}}\left|b_{j}\right| R^{-1 / m_{j}}\right) .
$$

Choosing $R \geq 2^{M} R_{0}$ we have $\lambda \leq \frac{1}{2}$ and hence

$$
U_{j} \subset D\left(a_{j}, 2\left|b_{j}\right| R^{-1 / M}\right),
$$

provided $j$ is so large that $m_{j} \leq M$. Combining (3.1) and (3.3) we thus have

$$
D\left(a_{j}, \frac{1}{4 R}\left|b_{j}\right|\right) \subset U_{j} \subset D\left(a_{j}, 2 R^{-1 / M}\left|b_{j}\right|\right)
$$

for large $j$. Combining (3.2) and (3.3) we see that

$$
U_{j} \subset D\left(a_{j}, 8 R_{0}\left|a_{j}\right| R^{-1 / M}\right) .
$$

Choosing $R \geq\left(16 R_{0}\right)^{M}$ we thus have

$$
U_{j} \subset D\left(a_{j}, \frac{1}{2}\left|a_{j}\right|\right) \subset D\left(0, \frac{3}{2}\left|a_{j}\right|\right) .
$$

Next we note that the $U_{j}$ are pairwise disjoint. Combining this with (3.1) and (3.4) we see that if $n(r)$ denotes the number of $a_{j}$ contained in the closed disc $\overline{D(0, r)}$, then

$$
\begin{aligned}
\frac{\pi}{16 R^{2}} \sum_{j=1}^{n(r)}\left|b_{j}\right|^{2} & =\operatorname{area}\left(\bigcup_{j=1}^{n(r)} D\left(a_{j}, \frac{1}{4 R}\left|b_{j}\right|\right)\right) \\
& \leq \operatorname{area}\left(\bigcup_{j=1}^{n(r)} U_{j}\right) \leq \operatorname{area}\left(D\left(0, \frac{3}{2} r\right)\right)=\frac{9 \pi}{4} r^{2} .
\end{aligned}
$$

Hence

$$
\sum_{j=1}^{n(r)}\left|b_{j}\right|^{2} \leq 36 R^{2} r^{2} .
$$


We shall use (3.5) to prove the following result.

Lemma 3.1. If

then

$$
t>\frac{2 M \rho}{2+M \rho}
$$

$$
\sum_{j=1}^{\infty}\left(\frac{\left|b_{j}\right|}{\left|a_{j}\right|^{1+1 / M}}\right)^{t}<\infty
$$

Proof. We put

$$
s=\frac{\rho}{2}\left(\frac{t}{2}-1\right)+1+\frac{t}{2 M}
$$

Then

$$
s>\frac{\rho}{2}\left(\frac{M \rho}{2+M \rho}-1\right)+1+\frac{\rho}{2+M \rho}=1 .
$$

For $l \geq 0$ we put

$$
P(l)=\left\{j \in \mathbb{N}: n\left(2^{l}\right) \leq j<n\left(2^{l+1}\right)\right\}=\left\{j \in \mathbb{N}: 2^{l} \leq\left|a_{j}\right|<2^{l+1}\right\}
$$

and

$$
S_{l}=\sum_{j \in P(l)}\left(\frac{\left|b_{j}\right|}{\left|a_{j}\right|^{1+1 / M}}\right)^{t}=\sum_{j \in P(l)}\left(\frac{\left|b_{j}\right|}{\left|a_{j}\right|^{s}}\right)^{t}\left(\frac{1}{\left|a_{j}\right|}\right)^{t(1-s+1 / M)} .
$$

We now apply Hölder's inequality, with $p=2 / t$ and $q=2 /(2-t)$. Putting

$$
\alpha=t\left(1-s+\frac{1}{M}\right) \frac{2}{2-t}=t \frac{2+M \rho}{2 M}>\rho
$$

we obtain

$$
S_{l} \leq\left(\sum_{j \in P(l)} \frac{\left|b_{j}\right|^{2}}{\left|a_{j}\right|^{2 s}}\right)^{t / 2}\left(\sum_{j \in P(l)} \frac{1}{\left|a_{j}\right|^{\alpha}}\right)^{(2-t) / 2} .
$$

Since $\alpha>\rho$ the series $\sum_{j=1}^{\infty}\left|a_{j}\right|^{-\alpha}$ converges by Lemma 2.4. Thus

$$
\left(\sum_{j \in P(l)} \frac{1}{\left|a_{j}\right|^{\alpha}}\right)^{(2-t) / 2} \leq A:=\left(\sum_{j=1}^{\infty} \frac{1}{\left|a_{j}\right|^{\alpha}}\right)^{(2-t) / 2}<\infty .
$$

We now see, using (3.5), that

$$
\begin{aligned}
S_{l} & \leq A\left(\sum_{j \in P(l)} \frac{\left|b_{j}\right|^{2}}{\left|a_{j}\right|^{2 s}}\right)^{t / 2} \leq A\left(\frac{1}{\left(2^{l}\right)^{2 s}} \sum_{j \in P(l)}\left|b_{j}\right|^{2}\right)^{t / 2} \\
& \leq \frac{A}{2^{l s t}}\left(36 R^{2} 2^{2(l+1)}\right)^{t / 2}=A(12 R)^{t}\left(2^{t(1-s)}\right)^{l} .
\end{aligned}
$$

Since $t(1-s)<0$, the series $\sum_{l=0}^{\infty} S_{l}$ converges.

Continuing with the proof of Theorem 1.1 we note that in each simply connected domain $D \subset B(R) \backslash\{\infty\}$ we can define all branches of the inverse function of $f$. Let $g_{j}$ be a branch of $f^{-1}$ that maps $D$ to $U_{j}$. Thus

$$
g_{j}(z)=\psi_{j}\left(\frac{1}{z^{1 / m_{j}}}\right)
$$


for some branch of the $m_{j}$-th root. We obtain

$$
g_{j}^{\prime}(z)=-\psi_{j}^{\prime}\left(\frac{1}{z^{1 / m_{j}}}\right) \frac{1}{m_{j} z^{1+1 / m_{j}}} .
$$

Since we assumed that $R \geq 2^{M} R_{0}$ we deduce from (2.2) with $\lambda=\frac{1}{2}$ that

$$
\left|g_{j}^{\prime}(z)\right| \leq \frac{12\left|\psi_{j}^{\prime}(0)\right|}{M|z|^{1+1 / M}}=\frac{12\left|b_{j}\right|}{M|z|^{1+1 / M}} \leq \frac{12\left|b_{j}\right|}{|z|^{1+1 / M}}
$$

for $z \in D \subset B(R) \backslash\{\infty\}$, provided $j$ is so large that $m_{j} \leq M$. From (3.3) we deduce that

$$
\operatorname{diam}\left(U_{k}\right) \leq \frac{4}{R^{1 / M}}\left|b_{k}\right|
$$

Moreover, if $U_{j} \subset B(R)$, then

$$
\begin{aligned}
\operatorname{diam} g_{j}\left(U_{k}\right) & \leq \sup _{z \in U_{k}}\left|g_{j}^{\prime}(z)\right| \operatorname{diam} U_{k} \\
& \leq \frac{12\left|b_{j}\right|}{\left(\frac{1}{2}\left|a_{k}\right|\right)^{1+1 / M}} \frac{4}{R^{1 / M}}\left|b_{k}\right|=2^{1 / M} 24 \frac{4}{R^{1 / M}}\left|b_{j}\right| \frac{\left|b_{k}\right|}{\left|a_{k}\right|^{1+1 / M}} .
\end{aligned}
$$

Induction shows that if $U_{j_{1}}, U_{j_{2}}, \ldots, U_{j_{l}} \subset B(R)$, then

$$
\begin{aligned}
& \operatorname{diam}\left(\left(g_{j_{1}} \circ g_{j_{2}} \circ \ldots \circ g_{j_{l-1}}\right)\left(U_{j_{l}}\right)\right) \\
\leq & \left(2^{1 / M} 24\right)^{l-1} \frac{4}{R^{1 / M}}\left|b_{j_{1}}\right| \frac{\left|b_{j_{2}}\right|}{\left|a_{j_{2}}\right|^{1+1 / M}} \cdots \frac{\left|b_{j_{l}}\right|}{\left|a_{j_{l}}\right|^{1+1 / M}} .
\end{aligned}
$$

In order to obtain an estimate for the spherical diameter, we estimate the spherical distance $\chi\left(z_{1}, z_{2}\right)$ of two points $z_{1}, z_{2} \in D\left(a_{j}, \frac{1}{2}\left|a_{j}\right|\right)$. We have

$$
\chi\left(z_{1}, z_{2}\right)=\frac{2\left|z_{1}-z_{2}\right|}{\sqrt{1+\left|z_{1}\right|^{2}} \sqrt{1+\left|z_{2}\right|^{2}}} \leq \frac{2\left|z_{1}-z_{2}\right|}{1+\frac{1}{4}\left|a_{j}\right|^{2}} \leq \frac{8\left|z_{1}-z_{2}\right|}{1+\left|a_{j}\right|^{2}} \leq \frac{8\left|z_{1}-z_{2}\right|}{\left|a_{j}\right|^{1+1 / M}} .
$$

Thus

$$
\operatorname{diam}_{\chi}(K) \leq \frac{8}{\left|a_{j}\right|^{1+1 / M}} \operatorname{diam}(K)
$$

for $K \subset U_{j}$ and hence (3.9) yields

$$
\operatorname{diam}_{\chi}\left(\left(g_{j_{1}} \circ g_{j_{2}} \circ \ldots \circ g_{j_{l-1}}\right)\left(U_{j_{l}}\right)\right) \leq\left(2^{1 / M} 24\right)^{l-1} \frac{32}{R^{1 / M}} \prod_{k=1}^{l} \frac{\left|b_{j_{k}}\right|}{\left|a_{j_{k}}\right|^{1+1 / M}}
$$

Now there are $m_{j_{k}}$ branches of the inverse function of $f$ mapping $U_{j_{k+1}}$ into $U_{j_{k}}$, for $k=1,2, \ldots, l-1$. Overall we see that there are

$$
\prod_{k=1}^{l-1} m_{j_{k}} \leq M^{l-1}
$$

sets of diameters bounded as in (3.10) which cover all those components $V$ of $f^{-l}(B(R))$ for which $f^{k}(V) \subset U_{j_{k+1}} \subset B(R)$ for $k=0,1, \ldots, l-1$. We denote by $E_{l}$ the collection of all components $V$ of $f^{-l}(B(R))$ for which $f^{k}(V) \subset B(R)$ for $k=0,1, \ldots, l-1$.

Next we note that (3.4) implies that if $U_{j} \cap B(3 R) \neq \emptyset$, then $\left|a_{j}\right|>2 R$ and $U_{j} \subset B(R)$. We conclude that $E_{l}$ is a cover of the set

$$
\left\{z \in B(3 R): f^{k}(z) \in B(3 R) \text { for } 1 \leq k \leq l-1\right\} .
$$


Moreover, if $t>2 M \rho /(2+M \rho)$, then

$$
\begin{aligned}
& \sum_{V \in E_{l}}\left(\operatorname{diam}_{\chi}(V)\right)^{t} \\
& \quad \leq M^{l-1}\left(\left(2^{1 / M} 24\right)^{l-1} \frac{32}{R^{1 / M}}\right)^{t} \sum_{j_{1}=n(R)}^{\infty} \ldots \sum_{j_{l}=n(R)}^{\infty} \prod_{k=1}^{l}\left(\frac{\left|b_{j_{k}}\right|}{\left|a_{j_{k}}\right|^{1+1 / M}}\right)^{t} \\
& \quad=\frac{1}{M}\left(\frac{32}{(2 R)^{1 / M} 24}\right)^{t}\left(M\left(2^{1 / M} 24\right)^{t} \sum_{j=n(R)}^{\infty}\left(\frac{\left|b_{j}\right|}{\left|a_{j}\right|^{1+1 / M}}\right)^{t}\right)^{l} .
\end{aligned}
$$

Lemma 3.1 implies that

$$
M\left(2^{1 / M} 24\right)^{t} \sum_{j=n(R)}^{\infty}\left(\frac{\left|b_{j}\right|}{\left|a_{j}\right|^{1+1 / M}}\right)^{t}<1
$$

for large $R$. For such an $R$ we find that

$$
\lim _{l \rightarrow \infty} \sum_{V \in E_{l}}\left(\operatorname{diam}_{\chi}(V)\right)^{t}=0
$$

and thus

$$
\mathrm{HD}\left(\left\{z \in B(3 R): f^{k}(z) \in B(3 R) \text { for all } k \in \mathbb{N}\right\}\right) \leq t .
$$

Hence $\operatorname{HD}\left(I_{3 R}(f)\right) \leq t$. As $t>2 M \rho /(2+M \rho)$ was arbitrary, the conclusion follows.

\section{LOWER BOUNDS FOR THE HAUSDORFF DIMENSION}

In order to prove Theorem [1.2, we shall use results of Mayer [17] and McMullen [18. For measurable subsets $X, Y$ of the plane (or sphere) we define the Euclidean and the spherical density of $X$ in $Y$ by

$$
\operatorname{dens}(X, Y)=\frac{\operatorname{area}(X \cap Y)}{\operatorname{area}(Y)} \text { and } \operatorname{dens}_{\chi}(X, Y)=\frac{\operatorname{area}_{\chi}(X \cap Y)}{\operatorname{area}_{\chi}(Y)} \text {. }
$$

Note that if

$$
Y \subset\{z \in \mathbb{C}: R<|z|<S\}
$$

then

$$
\frac{4}{\left(1+S^{2}\right)^{2}} \operatorname{area}(Y) \leq \operatorname{area}_{\chi}(Y) \leq \frac{4}{\left(1+R^{2}\right)^{2}} \operatorname{area}(Y)
$$

and thus

$$
\left(\frac{1+R^{2}}{1+S^{2}}\right)^{2} \operatorname{dens}(X, Y) \leq \operatorname{dens}_{\chi}(X, Y) \leq\left(\frac{1+S^{2}}{1+R^{2}}\right)^{2} \operatorname{dens}(X, Y)
$$

if $Y$ satisfies (4.1).

In order to state McMullen's result, consider for $l \in \mathbb{N}$ a collection $E_{l}$ of disjoint compact subsets of $\widehat{\mathbb{C}}$ such that the following two conditions are satisfied:

(a) every element of $E_{l+1}$ is contained in a unique element of $E_{l}$;

(b) every element of $E_{l}$ contains at least one element of $E_{l+1}$. 
Denote by $\bar{E}_{l}$ the union of all elements of $E_{l}$ and put $E=\bigcap_{l=1}^{\infty} \bar{E}_{l}$. Suppose that $\left(\Delta_{l}\right)$ and $\left(d_{l}\right)$ are sequences of positive real numbers such that if $V \in E_{l}$, then

$$
\operatorname{dens}_{\chi}\left(\bar{E}_{l+1}, V\right) \geq \Delta_{l}
$$

and

$$
\operatorname{diam}_{\chi}(V) \leq d_{l} .
$$

Then we have the following result [18].

Lemma 4.1. Let $E, E_{l}, \Delta_{l}$ and $d_{l}$ be as above. Then

$$
\limsup _{l \rightarrow \infty} \frac{\sum_{j=1}^{l+1}\left|\log \Delta_{j}\right|}{\left|\log d_{l}\right|} \geq 2-\operatorname{dim} E .
$$

We remark that McMullen worked with the Euclidean density, but the above lemma follows directly from his result.

We shall use Lemma 4.1 to prove (1.3). Of course, it follows from (1.3) that

$$
\operatorname{HD}\left(I_{R}(f)\right) \geq \frac{2 M \rho}{2+M \rho},
$$

for all $R>0$, but the application of Lemma 4.1 does not seem to yield (1.4), which says that we have strict inequality in (4.3). However, in order to illustrate the method, we shall first use Lemma 4.1 to prove (4.3). We will then describe the modifications that have to be made in order to prove (1.3).

The proof of (1.4) is based on the following result due to Mayer [17, which he obtained using the theory of infinite iterated function systems developed by Mauldin and Urbański [16,

Lemma 4.2. Let $f$ be a transcendental meromorphic function with $\rho=\rho(f)<\infty$. Suppose that $f$ has a pole $c \in \mathbb{C} \backslash \overline{\operatorname{sing}\left(f^{-1}\right)}$ and denote by $M$ the multiplicity of c. Suppose also that there are a neighbourhood $D$ of $c$ and constants $K>0$ and $\alpha>-1-1 / M$ such that $\left|f^{\prime}(z)\right| \leq K|z|^{\alpha}$ for $z \in f^{-1}(D)$. Then

$$
\operatorname{HD}(J(f)) \geq \frac{\rho}{\alpha+1+1 / M} .
$$

Actually Mayer [17, Remark 3.2] points out that if $\left(z_{n}\right)$ denotes the sequence of points where $f$ takes the value $c$ and if

$$
\sum_{n=1}^{\infty}\left|z_{n}\right|^{-\rho}
$$

diverges, then we have strict inequality in (4.4). Moreover, his proof shows that if $f$ has infinitely many poles $c$ which satisfy the hypothesis of Lemma 4.2 and for which the series (4.5) diverges, then

$$
\operatorname{HD}\left(I_{R}(f)\right)>\frac{\rho}{\alpha+1+1 / M}
$$

for each $R>0$. 


\section{Construction of the example}

In order to construct a function $f$ to which the results of the previous section can be applied we put $\mu=2 / \rho$ and define

$$
g(z)=2 \sum_{k=1}^{\infty} \frac{k^{\mu k} z^{k}}{z^{2 k}-k^{2 \mu k}}
$$

We note that if $k \geq(2|z|)^{1 / \mu}$, then

$$
\left|\frac{k^{\mu k} z^{k}}{z^{2 k}-k^{2 \mu k}}\right| \leq \frac{k^{\mu k}|z|^{k}}{k^{2 \mu k}-|z|^{2 k}} \leq 2 \frac{|z|^{k}}{k^{\mu k}} \leq 2^{1-k}
$$

Thus the series in (5.1) converges locally uniformly and hence it defines a function $g$ meromorphic in $\mathbb{C}$. The poles of $g$ are at the points

$$
u_{k, l}=k^{\mu} \exp (\pi i l / k)
$$

where $k \in \mathbb{N}$ and $0 \leq l \leq 2 k-1$. With $v_{k, l}=k^{\mu-1} \exp (\pi i l(1-k) / k)$ we have

$$
g(z)=\sum_{k=1}^{\infty} \sum_{l=0}^{2 k-1} \frac{v_{k, l}}{z-u_{k, l}}
$$

Note that

$$
\left|v_{k, l}\right|=k^{\mu-1}=\left|u_{k, l}\right|^{1-1 / \mu}=\left|u_{k, l}\right|^{1-\rho / 2} .
$$

We will show that $g$ is bounded on the 'spider's web' $W=W_{1} \cup W_{2}$ where

$$
W_{1}=\bigcup_{n \geq 1}\left\{z:|z|=\left(n+\frac{1}{2}\right)^{\mu}\right\}
$$

and

$$
W_{2}=\bigcup_{n \geq 2}\left\{r e^{i \pi(2 m-1) / 2 n}:\left(n-\frac{1}{2}\right)^{\mu} \leq r \leq\left(n+\frac{1}{2}\right)^{\mu}, 1 \leq m \leq 2 n\right\} .
$$

First let $z \in W_{1}$, say $|z|=\left(n+\frac{1}{2}\right)^{\mu}$ where $n \in \mathbb{N}$. Then

$$
\begin{aligned}
\frac{1}{2}|g(z)| & \leq \sum_{k=1}^{n} \frac{k^{\mu k}|z|^{k}}{|z|^{2 k}-k^{2 \mu k}}+\sum_{k=n+1}^{\infty} \frac{k^{\mu k}|z|^{k}}{k^{2 \mu k}-|z|^{2 k}} \\
& =\sum_{k=1}^{n} \frac{k^{\mu k}}{|z|^{k}-k^{\mu k}} \frac{|z|^{k}}{|z|^{k}+k^{\mu k}}+\sum_{k=n+1}^{\infty} \frac{k^{\mu k}}{k^{\mu k}+|z|^{k}} \frac{|z|^{k}}{k^{\mu k}-|z|^{k}} \\
& \leq \sum_{k=1}^{n} \frac{k^{\mu k}}{|z|^{k}-k^{\mu k}}+\sum_{k=n+1}^{\infty} \frac{|z|^{k}}{k^{\mu k}-|z|^{k}} \\
& =\sum_{k=1}^{n} \frac{1}{\left(\frac{n+\frac{1}{2}}{k}\right)^{\mu k}-1}+\sum_{k=n+1}^{\infty} \frac{1}{\left(\frac{k}{n+\frac{1}{2}}\right)^{\mu k}-1} \\
& =\Sigma_{1, n}+\Sigma_{2, n} .
\end{aligned}
$$


Since $\log x \geq(x-1) \log 2$ for $1 \leq x \leq 2$ we see that if $\frac{n}{2}<k \leq n$, then

$$
\begin{aligned}
\left(\frac{n+\frac{1}{2}}{k}\right)^{\mu k} & =\exp \left(\mu k \log \left(\frac{n+\frac{1}{2}}{k}\right)\right) \\
& \geq \exp \left(\mu k \frac{n+\frac{1}{2}-k}{k} \log 2\right)=2^{\mu\left(n+\frac{1}{2}-k\right)}
\end{aligned}
$$

With $l=n+1-k$ we deduce that

$$
\begin{aligned}
\Sigma_{1, n} & \leq \sum_{k=1}^{\left[\frac{n}{2}\right]} \frac{1}{2^{\mu k}-1}+\sum_{k=\left[\frac{n}{2}\right]+1}^{n} \frac{1}{2^{\mu\left(n+\frac{1}{2}-k\right)}-1} \\
& =\sum_{k=1}^{\left[\frac{n}{2}\right]} \frac{1}{2^{\mu k}-1}+\sum_{l=1}^{n-\left[\frac{n}{2}\right]} \frac{1}{2^{\mu\left(l-\frac{1}{2}\right)}-1} \\
& \leq \sum_{k=1}^{\infty} \frac{1}{2^{\mu k}-1}+\sum_{l=1}^{\infty} \frac{1}{2^{\mu\left(l-\frac{1}{2}\right)}-1}=: C .
\end{aligned}
$$

Similarly we obtain

$$
\Sigma_{2, n} \leq \sum_{k=n+1}^{2 n} \frac{1}{\left(\frac{k}{n+\frac{1}{2}}\right)^{\mu k}-1}+\sum_{k=2 n+1}^{\infty} \frac{1}{2^{\mu k}-1} .
$$

We note that if $n+1 \leq k \leq 2 n$, then

$$
\begin{aligned}
\left(\frac{k}{n+\frac{1}{2}}\right)^{\mu k} & =\exp \left(\mu k \log \left(\frac{k}{n+\frac{1}{2}}\right)\right) \\
& \geq \exp \left(\mu k\left(\frac{k-n-\frac{1}{2}}{n+\frac{1}{2}}\right) \log 2\right) \\
& \geq \exp \left(\mu\left(k-n-\frac{1}{2}\right) \log 2\right)=2^{\mu\left(k-n-\frac{1}{2}\right)}
\end{aligned}
$$

With $l=k-n$ we obtain

$$
\Sigma_{2, n} \leq \sum_{l=1}^{n} \frac{1}{2^{\mu\left(l-\frac{1}{2}\right)}-1}+\sum_{k=2 n+1}^{\infty} \frac{1}{2^{\mu k}-1} \leq C .
$$

Combining (5.3) with (5.4) we find that

$$
|g(z)| \leq 4 C \text { for }|z|=\left(n+\frac{1}{2}\right)^{\mu} .
$$

Now let $z \in W_{2}$, say $z=r e^{i \pi(2 m-1) / 2 n}$, where $\left(n-\frac{1}{2}\right)^{\mu} \leq r \leq\left(n+\frac{1}{2}\right)^{\mu}$ and $1 \leq m \leq 2 n$. Then $z^{2 n}=-r^{2 n}$ and hence

$$
\left|\frac{n^{\mu n} r^{n}}{z^{2 n}-n^{2 \mu n}}\right|=\frac{n^{\mu n} r^{n}}{r^{2 n}+n^{2 \mu n}} .
$$


Similar estimates as above now yield

$$
\begin{aligned}
\frac{1}{2}|g(z)| & \leq \sum_{k=1}^{n-1} \frac{k^{\mu k} r^{k}}{r^{2 k}-k^{2 \mu k}}+\frac{n^{\mu n} r^{n}}{r^{2 n}+n^{2 \mu k}}+\sum_{k=n+1}^{\infty} \frac{k^{\mu k} r^{k}}{k^{2 \mu k}-r^{2 k}} \\
& \leq \sum_{k=1}^{n} \frac{k^{\mu k}}{\left(n-\frac{1}{2}\right)^{\mu k}-k^{\mu k}}+2+\sum_{k=n+1}^{\infty} \frac{\left(n+\frac{1}{2}\right)^{\mu k}}{k^{\mu k}-\left(n+\frac{1}{2}\right)^{k}} \\
& =\Sigma_{1, n-1}+2+\Sigma_{2, n} \leq 2 C+2 .
\end{aligned}
$$

We obtain

$$
|g(z)| \leq 4 C+4 \text { for } z \in W .
$$

Next we want to show that $g$ is actually bounded on a larger set. To do this we note that

$$
\left(n+\frac{1}{2}\right)^{\mu}-n^{\mu} \sim n^{\mu}-\left(n-\frac{1}{2}\right)^{\mu} \sim \frac{\mu}{2} n^{\mu-1}
$$

and

$$
\left|u_{n, m}-u_{n, m+1}\right|=n^{\mu}\left|e^{i \pi / n}-1\right| \sim \pi n^{\mu-1}
$$

as $n \rightarrow \infty$. It follows that there exists $\eta>0$ such that if $W_{n, m}$ denotes the component of $\mathbb{C} \backslash W$ that contains $u_{n, m}$, then

$$
\operatorname{dist}\left(u_{n, m}, \partial W_{n, m}\right) \geq 2 \eta n^{\mu-1}
$$

for all $n \in \mathbb{N}$ and $m \in\{0,1, \ldots, 2 n-1\}$. The function

$$
h(z)=g(z)-\frac{v_{n, m}}{z-u_{n, m}}
$$

is holomorphic in the closure of $W_{n, m}$ and for $z \in \partial W_{n, m}$ we have

$$
|h(z)| \leq|g(z)|+\frac{v_{n, m}}{\left|z-u_{n, m}\right|} \leq 4 C+4+\frac{n^{\mu-1}}{2 \eta n^{\mu-1}}=4 C+4+\frac{1}{2 \eta} .
$$

By the maximum principle,

$$
|h(z)| \leq 4 C+4+\frac{1}{2 \eta} \quad \text { for } \quad z \in W_{n, m} .
$$

We put $r_{n}=\eta n^{\mu-1}$ and deduce that if $z \in W_{n, m} \backslash D\left(u_{n, m}, r_{n}\right)$, then

$$
|g(z)| \leq|h(z)|+\frac{\left|v_{n, m}\right|}{r_{n}} \leq 4 C+4+\frac{3}{2 \eta} .
$$

We find that $g$ is large only in small neighbourhoods of the poles.

On the other hand, we will show that sufficiently small neighbourhoods of the poles do not contain critical points of $g$. This will then imply that the set of critical values of $g$ is bounded so that $g \in \mathcal{B}$.

In order to estimate the distance of the critical points to the poles, let $z \in \partial W_{n, m}$. If $n^{\prime}, m^{\prime}$ are such that also $z \in \partial W_{n^{\prime}, m^{\prime}}$, then $\left|n-n^{\prime}\right| \leq 1$. Thus $r_{n} \leq 2 r_{n^{\prime}}$ and hence $\partial D\left(z, \frac{1}{2} r_{n}\right) \cap D\left(u_{n^{\prime}, m^{\prime}}, r_{n^{\prime}}\right)=\emptyset$. It follows that

$$
\left|g^{\prime}(z)\right|=\frac{1}{2 \pi}\left|\int_{|\zeta-z|=\frac{1}{2} r_{n}} \frac{g(\zeta)}{(\zeta-z)^{2}} d \zeta\right| \leq \frac{4}{r_{n}} \max _{|\zeta-z|=\frac{1}{2} r_{n}}|g(z)| \leq \frac{4}{r_{n}}\left(4 C+4+\frac{3}{2 \eta}\right) .
$$


This implies that if $z \in \partial W_{n, m}$, then

$$
\left|h^{\prime}(z)\right| \leq\left|g^{\prime}(z)\right|+\frac{\left|v_{n . m}\right|}{\left|z-u_{n, m}\right|^{2}} \leq \frac{4}{r_{n}}\left(4 C+4+\frac{2}{\eta}\right) .
$$

Again we have

$$
\left|h^{\prime}(z)\right| \leq \frac{4}{r_{n}}\left(4 C+4+\frac{2}{\eta}\right) \quad \text { for } \quad z \in W_{n, m}
$$

by the maximum principle. We deduce that if $\delta>0$ is chosen sufficiently small and $z \in D\left(u_{n, m}, \delta r_{n}\right)$, then

$$
\left|g^{\prime}(z)\right| \geq \frac{\left|v_{n, m}\right|}{\left|z-u_{n, m}\right|^{2}}-\left|h^{\prime}(z)\right| \geq \frac{4}{r_{n}}\left(\frac{1}{4 \delta^{2} \eta}-4 C-4-\frac{2}{\eta}\right)>0 .
$$

It follows that if $g^{\prime}(z)=0$ for some $z \in W_{n, m}$, then $\left|z-u_{n, m}\right| \geq \delta r_{n}$ and thus

$$
|g(z)| \leq|h(z)|+\frac{\left|v_{n, m}\right|}{\left|z-u_{n, m}\right|} \leq 4 C+4+\frac{1}{2 \eta}+\frac{1}{\delta \eta} .
$$

This implies that the set of critical values of $g$ is bounded. By (5.5) the same is true for the set of asymptotic values of $g$. Hence $g \in \mathcal{B}$.

To compute the order of $g$ we note that the number $n(r, g)$ of poles of $g$ in $\overline{D(0, r)}$ satisfies

$$
n(r, g)=\sum_{k=1}^{\left[r^{1 / \mu}\right]} 2 k \sim \int_{0}^{r^{1 / \mu}} 2 t d t=r^{2 / \mu}=r^{\rho}
$$

as $r \rightarrow \infty$. Thus

$$
N(r, g)=\int_{0}^{r} \frac{n(t, g)}{t} d t \sim \frac{1}{\rho} r^{\rho} .
$$

By (5.5) we have $m(r, g) \leq 4 C+4$ if $r$ has the form $r=\left(k+\frac{1}{2}\right)^{\mu}$ for some $k \in \mathbb{N}$. It follows that

$$
T(r, g)=N(r, g)+m(r, g) \sim \frac{1}{\rho} r^{\rho}
$$

as $r \rightarrow \infty$ through $r$-values of the form $r=\left(k+\frac{1}{2}\right)^{\mu}$. But since $T(r, g)$ is an increasing function of $r$, the relation (5.8) actually holds for all values of $r$. Hence $g$ has order $\rho$.

We now put

$$
f(z)=g(z)^{M} .
$$

It follows that $f \in \mathcal{B}$ and that $f$ has order $\rho$.

\section{Proof of Theorem 1.2}

Let $f$ be the function constructed in section 5 . As in section 3 we denote the sequence of poles by $\left(a_{j}\right)$, ordered such that $\left|a_{j}\right| \leq\left|a_{j+1}\right|$ for all $j \in \mathbb{N}$. For each $j \in \mathbb{N}$ we thus have $a_{j}=u_{n, m}$ for some $n \in \mathbb{N}$ and $0 \leq m \leq 2 n-1$. It is not difficult to see that $j \sim n^{2}$ as $j \rightarrow \infty$ if $a_{j}=u_{n, m}$. Hence $\left|a_{j}\right|=\left|u_{n, m}\right|=n^{\mu} \sim j^{\mu / 2}=j^{1 / \rho}$ as $j \rightarrow \infty$. Choose $b_{j}$ as in section 3 so that

$$
f(z) \sim\left(\frac{b_{j}}{z-a_{j}}\right)^{M} \quad \text { as } \quad z \rightarrow a_{j} .
$$


Then $b_{j}=v_{n, m}$ if $a_{j}=u_{n, m}$ and hence

$$
\left|b_{j}\right|=\left|a_{j}\right|^{1-\rho / 2}
$$

by (5.2). Choose $R>0$ large and let $E_{l}$ be as in section 3 . Thus $E_{l}$ consists of all components $V$ of $f^{-l}(B(R))$ for which $f^{k}(V) \subset B(R)$ for $0 \leq k \leq l-1$. Clearly $E=\bigcap_{l=1}^{\infty} \bar{E}_{l} \subset I_{R}(f)$.

We deduce from (3.10) that if $V \in E_{l}$ such that $f^{k}(V) \subset U_{j_{k+1}}$ for $0 \leq k \leq l-1$, then

$$
\operatorname{diam}_{\chi}(V) \leq\left(2^{1 / M} 24\right)^{l-1} \frac{32}{R^{1 / M}} \prod_{k=1}^{l} \frac{\left|b_{j_{k}}\right|}{\left|a_{j_{k}}\right|^{1+1 / M}} .
$$

By (6.1) we have

$$
\frac{\left|b_{j_{k}}\right|}{\left|a_{j_{k}}\right|^{1+1 / M}}=\frac{1}{\left|a_{j_{k}}\right|^{\rho / 2+1 / M}}
$$

and since $\left|a_{j_{k}}\right|>R$ we obtain

$$
\operatorname{diam}_{\chi}(V) \leq\left(\frac{A}{R^{\rho / 2+1 / M}}\right)^{l}
$$

for some constant $A>0$ if $V \in E_{l}$. Thus we can apply Lemma 4.1 with

$$
d_{l}=\left(\frac{A}{R^{\rho / 2+1 / M}}\right)^{l} .
$$

In order to estimate $\Delta_{l}$ we note that

$$
W_{n, m} \subset D\left(u_{n, m},\left(\frac{\mu}{2}+\frac{\pi}{2}\right) n^{\mu-1}\right)
$$

for large $n$ by (5.6) and (5.7). By (5.2) we have

$$
\left|v_{n, m}\right|=\left|u_{n, m}\right|^{1-\rho / 2}=n^{\mu(1-\rho / 2)}=n^{\mu-1} .
$$

With $\tau=\mu / 2+\pi / 2$ we see that $W_{n, m} \subset D\left(u_{n, m}, \tau\left|v_{n, m}\right|\right)$ if $n$ is large. Thus

$$
W_{n, m} \subset D\left(a_{j}, \tau\left|b_{j}\right|\right)
$$

if $a_{j}=u_{n, m}$. On the other hand, it follows from (3.1) and (5.5) that

$$
D\left(a_{j}, \frac{1}{4 R^{1 / M}}\left|b_{j}\right|\right) \subset U_{j}=W_{n, m} \cap f^{-1}(B(R)),
$$

provided $R$ is large enough. We conclude that

$$
\operatorname{dens}\left(f^{-1}(B(R)), W_{n, m}\right) \geq \frac{1}{16 \tau^{2} R^{2 / M}} .
$$

Since

$$
W_{n, m} \subset\left\{z \in \mathbb{C}:\left(n-\frac{1}{2}\right)^{\mu} \leq|z| \leq\left(n+\frac{1}{2}\right)^{\mu}\right\}
$$

and since $\left(n+\frac{1}{2}\right)^{\mu} /\left(n-\frac{1}{2}\right)^{\mu} \rightarrow 1$ as $n \rightarrow \infty$ this implies that if $S \geq R$ and

$$
A(S)=\{z \in \mathbb{C}: S<|z|<2 S\},
$$

then

$$
\operatorname{dens}\left(\bar{E}_{1}, A(S)\right) \geq \frac{1}{17 \tau^{2} R^{2 / M}} .
$$


We now consider a branch $g_{j}$ of $f^{-1}$ which maps $A^{\prime}(S)=A(S) \backslash(-2 S,-S)$ into $U_{j}$. Recall that $g_{j}$ has the form (3.6). It follows from (3.8) that

$$
\left|g_{j}^{\prime}(z)\right| \leq \frac{12\left|b_{j}\right|}{M S^{1+1 / M}}
$$

for $z \in A^{\prime}(S)$. We obtained (3.8) from (3.7) by using the right inequality of Koebe's theorem (2.2). Using the left inequality instead we obtain

$$
\left|g_{j}^{\prime}(z)\right| \geq \frac{4\left|b_{j}\right|}{27 M|z|^{1+1 / M}} \geq \frac{4\left|b_{j}\right|}{27 M(2 S)^{1+1 / M}}
$$

for $z \in A^{\prime}(S)$. With $K=2^{1+1 / M} 81$ we obtain

$$
\sup _{u, v \in A^{\prime}(S)}\left|\frac{g_{j}^{\prime}(u)}{g_{j}^{\prime}(v)}\right| \leq K
$$

provided $S$ is large enough. We deduce that

$$
\text { dens }\left(g_{j}\left(\bar{E}_{1}\right), g_{j}\left(A^{\prime}(S)\right)\right) \geq \frac{1}{K^{2}} \operatorname{dens}\left(\bar{E}_{1}, A^{\prime}(S)\right) \geq \frac{1}{17 K^{2} \tau^{2} R^{2 / M}} .
$$

Applying this for all $S$ of the form $S=2^{k} R$ with $k \geq 0$ and for all branches $g_{j}$ mapping to $U_{j}$ we deduce that

$$
\operatorname{dens}\left(\bar{E}_{2}, U_{j}\right) \geq \frac{1}{17 K^{2} \tau^{2} R^{2 / M}}
$$

for each $U_{j}$ in $E_{1}$. Now let $V \in E_{l}$ and $j_{1}, j_{2}, \ldots, j_{k}$ be such that $f^{k}(V) \subset U_{j_{k+1}}$ for $0 \leq k \leq l-1$. Then $f^{l-1}(V)=U_{j_{l}}$ and

$$
f^{l-1}\left(\bar{E}_{l+1} \cap V\right)=\bar{E}_{2} \cap U_{j_{l}} .
$$

For large $R$ a branch of $f^{-1}$ that maps $U_{j_{l}}$ into $U_{j_{l-1}}$ extends univalently to $D\left(a_{j_{l}}, \frac{3}{4} a_{j_{l}}\right)$ and it maps $D\left(a_{j_{l}}, \frac{3}{4} a_{j_{l}}\right)$ into $B(R)$. Thus the branch of the inverse of $f^{l-1}$ which maps $U_{j_{l}}$ to $V$ extends univalently to $D\left(a_{j_{l}}, \frac{3}{4} a_{j_{l}}\right)$. Since $U_{j_{l}} \subset D\left(a_{j_{l}}, \frac{1}{2} a_{j_{l}}\right)$ by (3.4), we can now deduce from (6.4), (6.5) and Koebe's distortion theorem (2.2) with $\lambda=\frac{2}{3}$ that

$$
\operatorname{dens}\left(\bar{E}_{l+1}, V\right) \geq\left(\frac{1-\lambda}{1+\lambda}\right)^{4} \operatorname{dens}\left(\bar{E}_{2}, U_{j_{l}}\right) \geq\left(\frac{1-\lambda}{1+\lambda}\right)^{4} \frac{1}{17 K^{2} \tau^{2} R^{2 / M}} .
$$

Since $U_{j_{l}} \subset D\left(a_{j_{l}}, \frac{1}{2} a_{j_{l}}\right)$ we conclude using (4.2) that there exists a constant $B>0$ such that

$$
\operatorname{dens}_{\chi}\left(\bar{E}_{l+1}, V\right) \geq \frac{B}{R^{2 / M}} .
$$

Hence Lemma 4.1 can be applied with

$$
\Delta_{l}=\frac{B}{R^{2 / M}} .
$$

Using the values for $d_{l}$ and $\Delta_{l}$ given by (6.3) and (6.7) we find that

$$
\mathrm{HD}(E) \geq 2-\limsup _{l \rightarrow \infty} \frac{(l+1)\left(\log B-\frac{2}{M} \log R\right)}{l\left(\log A-\left(\frac{\rho}{2}+\frac{1}{M}\right) \log R\right)}=2-\frac{\log B-\frac{2}{M} \log R}{\log A-\left(\frac{\rho}{2}+\frac{1}{M}\right) \log R} .
$$


Since $E \subset I_{R}(f)$ and thus $\operatorname{HD}\left(I_{R}(f)\right) \geq \operatorname{HD}(E)$ and since $\operatorname{HD}\left(I_{R}(f)\right)$ is a nonincreasing function of $R$, we obtain

$$
\mathrm{HD}\left(I_{R}(f)\right) \geq 2-\limsup _{R \rightarrow \infty} \frac{\log B-\frac{2}{M} \log R}{\log A-\left(\frac{\rho}{2}+\frac{1}{M}\right) \log R}=2-\frac{\frac{2}{M}}{\frac{\rho}{2}+\frac{1}{M}}=\frac{2 M \rho}{2+M \rho} .
$$

Thus we have proved (4.3).

In order to prove (1.3) we choose a non-decreasing sequence $\left(R_{l}\right)$ which tends to $\infty$. We define $E_{l}$ as the set of all components $V$ of $f^{-l}\left(B\left(R_{l}\right)\right)$ which satisfy $f^{k}(V) \subset B\left(R_{k}\right)$ for $0 \leq k \leq l-1$. Then it follows that $E=\bigcap_{l=1}^{\infty} \bar{E}_{l} \subset I(f)$.

We now argue similarly as before. Instead of (6.2) and (6.6) we obtain

$$
\operatorname{diam}_{\chi}(V) \leq \prod_{k=1}^{l}\left(\frac{A}{R_{k}^{\rho / 2+1 / M}}\right) \quad \text { and } \quad \operatorname{dens}_{\chi}\left(\bar{E}_{l+1}, V\right) \geq \frac{B}{R_{l}^{2 / M}} .
$$

Thus we can apply Lemma 4.1 with

$$
d_{l}=A^{l} \prod_{k=1}^{l} \frac{1}{R_{k}^{\rho / 2+1 / M}} \quad \text { and } \quad \Delta_{l}=\frac{B}{R_{l}^{2 / M}} .
$$

We obtain

$$
\mathrm{HD}(E) \geq 2-\limsup _{l \rightarrow \infty} \frac{(l+1) \log B-\frac{2}{M} \sum_{k=1}^{l+1} \log R_{k}}{l \log A-\left(\frac{\rho}{2}+\frac{1}{M}\right) \sum_{k=1}^{l} \log R_{k}} .
$$

Choosing a sequence $\left(R_{l}\right)$ which does not tend to infinity too fast, for example $R_{k}=k$ for large $k$, we deduce that

$$
\mathrm{HD}(I(f)) \geq 2-\frac{\frac{2}{M}}{\frac{\rho}{2}+\frac{1}{M}}=\frac{2 M \rho}{2+M \rho} .
$$

The opposite inequality follows from Theorem 1.1. Thus we have proved (1.3).

To prove (1.4) we will now apply Lemma 4.2 and the remarks following it. Let $c$ be a pole of $f$ which has large modulus. Thus $c=u_{n, m}$, where $n$ is large and $0 \leq m \leq 2 n-1$. It follows from the consideration in section 3 that if $c$ is large enough, if $D$ is a sufficiently small neighbourhood of $c$ and if $z \in f^{-1}(D)$, then $z$ is in a small neighbourhood of one of the poles $u_{k, l}$. In particular, we can achieve that

$$
\frac{1}{2} \frac{\left|v_{k, l}\right|^{M}}{\left|z-u_{k, l}\right|^{M}} \leq|f(z)| \leq 2|c|
$$

and

$$
\left|f^{\prime}(z)\right| \leq 2 M \frac{\left|v_{k, l}\right|^{M}}{\left|z-u_{k, l}\right|^{M+1}}
$$

for some $k \in \mathbb{N}$ and $0 \leq l \leq 2 k-1$ if $|z|$ is sufficiently large. Combining (6.8) and (5.2) we see that

$$
\left|z-u_{k, l}\right| \geq\left(\frac{1}{4|c|}\right)^{1 / M}\left|v_{k, l}\right|=\left(\frac{1}{4|c|}\right)^{1 / M}\left|u_{k, l}\right|^{1-\rho / 2} .
$$

Now (6.8), (6.9) and (6.10) yield

$$
\left|f^{\prime}(z)\right| \leq 2 M \frac{\left|v_{k, l}\right|^{M}}{\left|z-u_{k, l}\right|^{M}} \frac{1}{\left|z-u_{k, l}\right|} \leq \frac{8 M|c|}{\left|z-u_{k, l}\right|} \leq 8 M|c|(4|c|)^{1 / M}\left|u_{k, l}\right|^{\rho / 2-1},
$$


and as $z$ is in a small neighbourhood of $u_{k, l}$ we obtain

$$
\left|f^{\prime}(z)\right| \leq K|z|^{\rho / 2-1}
$$

for some constant $K$. We can thus apply Lemma 4.2 with $\alpha=\rho / 2-1$ and obtain

$$
\mathrm{HD}(J(f)) \geq \frac{\rho}{\alpha+1+\frac{1}{M}}=\frac{\rho}{\frac{\rho}{2}+\frac{1}{M}}=\frac{2 M \rho}{2+M \rho} .
$$

As mentioned after Lemma 4.2, we can replace $J(f)$ by $I_{R}(f)$ here and thus we have again obtained (4.3).

However, we can do better. From (5.8) and the definition of $f$ we deduce that

$$
T(r, f) \sim \frac{M}{\rho} r^{\rho}
$$

as $r \rightarrow \infty$. Nevanlinna's second fundamental theorem implies that if $c_{1}, c_{2}, c_{3} \in \mathbb{C}$ are distinct, then

$$
\sum_{j=1}^{3} N\left(r, \frac{1}{f-c_{j}}\right) \geq(1-o(1)) T(r, f)
$$

as $r \rightarrow \infty$. We deduce that

$$
\int_{1}^{\infty} \frac{N\left(r, \frac{1}{f-c_{j}}\right)}{t^{\rho+1}} d t
$$

diverges for at least one value of $j$. The remarks before Lemma 2.4 now imply that the series (4.5) diverges for all $c \in \mathbb{C}$ with at most two exceptions. Hence (1.4) follows from (4.6).

Remark. Arguments similar to the ones used above show that with $p_{k}=[k \log k]$ the function

$$
f(z)=\sum_{k=2}^{\infty} \frac{(\log k)^{p_{k}} z^{p_{k}}}{z^{2 p_{k}}-(\log k)^{2 p_{k}}}
$$

is in $\mathcal{B}$, that $\infty$ is not an asymptotic value of $f$ and that $\operatorname{HD}(I(f))=2$. Clearly, $f$ has only simple poles.

To prove these assertions, note first that $f$ can be written in the form

$$
f(z)=\sum_{k=2}^{\infty} \sum_{l=0}^{2 p_{k}-1} \frac{v_{k, l}}{z-u_{k, l}},
$$

where

$$
u_{k, l}=(\log k) \exp \left(\pi i l / p_{k}\right)
$$

and

$$
\left|v_{k, l}\right|=\frac{\log k}{2 p_{k}} \sim \frac{1}{2 k}
$$

as $k \rightarrow \infty$. It turns out that $f$ is bounded on $W=W_{1} \cup W_{2}$ where

$$
W_{1}=\bigcup_{n \geq 1}\left\{z:|z|=\log \left(n+\frac{1}{2}\right)\right\}
$$

and

$$
W_{2}=\bigcup_{n \geq 2}\left\{r e^{i \pi(2 m-1) / 2 p_{k}}: \log \left(n-\frac{1}{2}\right) \leq r \leq \log \left(n+\frac{1}{2}\right), 1 \leq m \leq 2 p_{k}\right\} .
$$


Once this is known, it follows with $r_{n}=1 / n$ that if $\eta>0$, then $f$ is actually bounded on the set of all $z \in \mathbb{C}$ such that $\left|z-u_{n, m}\right| \geq \eta r_{n}$ for all $n$ and $m$. As above this can be used to show that $f \in \mathcal{B}$.

Instead of (6.1) we now find that

$$
\left|b_{j}\right| \sim \frac{1}{2} e^{-\left|a_{j}\right|}
$$

and instead of (6.2) we obtain

$$
\operatorname{diam}_{\chi}(V) \leq\left(\frac{A e^{-R}}{R^{2}}\right)^{l} \leq e^{-l R / 2}
$$

so that we may take $d_{l}=\exp (-l R / 2)$ if $R$ is large. The densities dens $\left(\bar{E}_{l+1}, V\right)$ can also be estimated as before and we may again take $\Delta_{l}=B / R^{2}$ as in (6.7).

Using the estimates for $d_{l}$ and $\Delta_{l}$ we deduce from Lemma 4.1 as above that

$$
\operatorname{HD}\left(I_{R}(f)\right) \geq 2-\limsup _{R \rightarrow \infty} \frac{\log B-2 \log R}{\log A-\frac{1}{2} R}=2 .
$$

As before we can repeat the arguments with $R$ replaced by a sequence $\left(R_{l}\right)$ which tends to infinity slowly. We then find that $\operatorname{HD}(I(f))=2$ as claimed.

\section{Proof of Theorem 1.3}

Put $I_{R}^{\prime}=\left\{z \in \mathbb{C}:\left|f^{k}(z)\right|>R\right.$ for all $\left.k \geq 0\right\}$. We shall show that area $\left(I_{R}^{\prime}\right)=0$ if $R$ is sufficiently large. This easily implies that area $\left(I_{R}\right)=0$.

We use the notation of section 3 and, in addition, denote by $U_{j}^{0}$ the component of $B\left(R_{0}\right)$ that contains $a_{j}$. Then $U_{j}^{0} \cap U_{k}^{0}=\emptyset$ for $j \neq k$. In particular, $U_{j}^{0} \cap U_{k}=\emptyset$ for $j \neq k$ if $R \geq R_{0}$. It follows that $I_{R}^{\prime} \cap U_{j}^{0} \subset U_{j}$. By (3.1) we have

$$
U_{j}^{0} \supset D\left(a_{j}, \frac{1}{4 R_{0}^{1 / M}}\left|b_{j}\right|\right)
$$

while (3.3) yields that

$$
U_{j} \subset D\left(a_{j}, \frac{2}{R^{1 / M}}\left|b_{j}\right|\right) .
$$

Now suppose that area $\left(I_{R}^{\prime}\right)>0$, let $\xi$ be a density point of $I_{R}^{\prime}$ and put $w_{l}=f^{l}(\xi)$ for $l \geq 0$. Then $w_{l} \in U_{j_{l}}$ for some $j_{l} \in \mathbb{N}$. Thus $\left|w_{l}-a_{j_{l}}\right| \leq 2\left|b_{j_{l}}\right| R^{-1 / M}$ so that

$$
D\left(w_{l}, \frac{1}{5 R_{0}^{1 / M}}\left|b_{j_{l}}\right|\right) \subset D\left(a_{j_{l}}, \frac{1}{4 R_{0}^{1 / M}}\left|b_{j_{l}}\right|\right)
$$

for large $R$. Thus

$$
I_{R}^{\prime} \cap D\left(w_{l}, \frac{1}{5 R_{0}^{1 / M}}\left|b_{j_{l}}\right|\right) \subset D\left(a_{j_{l}}, \frac{2}{R^{1 / M}}\left|b_{j_{l}}\right|\right),
$$

which implies that

$$
\operatorname{dens}\left(I_{R}^{\prime}, D\left(w_{l}, \frac{1}{5 R_{0}^{1 / M}}\left|b_{j_{l}}\right|\right)\right) \leq 100\left(\frac{R_{0}}{R}\right)^{2 / M} .
$$

Similarly as in section 3 we see that if $R_{0}$ is chosen large enough and if $\varphi_{l}$ denotes the branch of the inverse function of $f^{l}$ which maps $w_{l}$ to $\xi$, then $\varphi_{l}$ has an analytic 
continuation to $D\left(w_{l}, 2 R_{0}^{-1 / M}\left|b_{j_{l}}\right| / 5\right)$. Applying Koebe's distortion theorem (2.2) with $\lambda=\frac{1}{2}$ we conclude that

$$
\begin{aligned}
\operatorname{dens} & \left(I_{R}^{\prime}, \varphi_{l}\left(D\left(w_{l}, \frac{1}{5 R_{0}^{1 / M}}\left|b_{j_{l}}\right|\right)\right)\right) \\
& \leq\left(\frac{1+\lambda}{1-\lambda}\right)^{4} \operatorname{dens}\left(I_{R}^{\prime}, D\left(w_{l}, \frac{1}{\left.\left.5 R_{0}^{1 / M}\left|b_{j_{l}}\right|\right)\right)}\right.\right. \\
& \leq 8100\left(\frac{R_{0}}{R}\right)^{2 / M} .
\end{aligned}
$$

Koebe's theorems (2.3) and (2.1) also yield that

$$
\begin{aligned}
D\left(\xi, \frac{1}{20 R_{0}^{1 / M}}\left|b_{j_{l}} \varphi_{l}^{\prime}\left(w_{l}\right)\right|\right) & \subset \varphi_{l}\left(D\left(w_{l}, \frac{1}{5 R_{0}^{1 / M}}\left|b_{j_{l}}\right|\right)\right) \\
& \subset D\left(\xi, \frac{2}{5 R_{0}^{1 / M}}\left|b_{j_{l}} \varphi_{l}^{\prime}\left(w_{l}\right)\right|\right) .
\end{aligned}
$$

With $r_{l}=\frac{2}{5} R_{0}^{-1 / M}\left|b_{j_{l}} \varphi_{l}^{\prime}\left(w_{l}\right)\right|$ we conclude that

$$
\operatorname{dens}\left(I_{R}^{\prime},\left(D\left(\xi, r_{l}\right)\right)\right) \leq 64 \cdot 8100\left(\frac{R_{0}}{R}\right)^{2 / M} .
$$

Let $g_{k}$ be the branch of the inverse function of $f$ which maps $w_{k+1}$ to $w_{k}$. By (3.8) we have

$$
\left|g_{k}^{\prime}\left(w_{k+1}\right)\right|<\frac{12\left|b_{j_{k}}\right|}{\left|w_{k+1}\right|^{1+1 / M}}
$$

Using (3.2) and (3.4) we obtain

$$
\left|g_{k}^{\prime}\left(w_{k+1}\right)\right| \leq \frac{96 R_{0}\left|a_{j_{k}}\right|}{\left|w_{k+1}\right|^{1 / M}\left|a_{j_{k+1}}\right|} \leq \frac{96 R_{0}\left|a_{j_{k}}\right|}{R^{1 / M}\left|a_{j_{k+1}}\right|} \leq \frac{1}{2} \frac{\left|a_{j_{k}}\right|}{\left|a_{j_{k+1}}\right|}
$$

for large $R$. Since $\varphi_{l}=g_{l-1} \circ g_{l-2} \circ \ldots \circ g_{0}$ we deduce that

$$
\left|\varphi_{l}^{\prime}\left(w_{l}\right)\right| \leq\left(\frac{1}{2}\right)^{l} \frac{\left|a_{j_{0}}\right|}{\left|a_{j_{l}}\right|} .
$$

Using (3.2) again we find that

$$
r_{l}=\frac{2}{5 R_{0}^{1 / M}}\left|b_{j_{l}} \varphi_{l}^{\prime}\left(w_{l}\right)\right| \leq \frac{2}{5 R_{0}^{1 / M}}\left|b_{j_{l}}\right|\left(\frac{1}{2}\right)^{l} \frac{\left|a_{j_{0}}\right|}{\left|a_{j_{l}}\right|} \leq \frac{8}{5} R_{0}^{1-1 / M}\left|a_{j_{0}}\right|\left(\frac{1}{2}\right)^{l} .
$$

Thus $r_{l} \rightarrow 0$ as $l \rightarrow \infty$.

If $R$ is so large that the right hand side of (7.1) is less than 1, we obtain a contradiction to the assumption that $\xi$ is a point of density.

We note that the argument shows that in fact the set of all $z \in \mathbb{C}$ for which

$$
\limsup _{k \rightarrow \infty}\left|f^{k}(z)\right|>R
$$

has area zero for large $R$. 


\section{Proof of Theorem 1.4}

We want to construct a function $f \in \mathcal{B}$ for which $\infty$ is not an asymptotic value and the multiplicity of the poles is unbounded such that area $(I(f))>0$.

We begin by choosing a sequence of discs $D\left(a_{j}, r_{j}\right)$ of radius less than 1 which are contained in $\{z \in \mathbb{C}:|z|>2\}$ such that the complement

$$
A=\mathbb{C} \backslash \bigcup_{j=1}^{\infty} D\left(a_{j}, r_{j}\right)
$$

is small in a certain sense. More specifically, we choose the $D\left(a_{j}, r_{j}\right)$ such that with

$$
P_{n}=\left\{z \in \mathbb{C}: 2^{n} \leq|z|<2^{n+1}\right\}
$$

the following properties are satisfied:

$$
\begin{gathered}
\overline{D\left(a_{j}, r_{j}\right)} \cap \overline{D\left(a_{k}, r_{k}\right)}=\emptyset \text { for } j, k \in \mathbb{N}, j \neq k, \\
I_{n}:=\left\{j \in \mathbb{N}: P_{n} \cap D\left(a_{j}, r_{j}\right) \neq \emptyset\right\} \text { is finite for } n \in \mathbb{N}
\end{gathered}
$$

and

$$
\text { area }\left(A \cap P_{n}\right)<1 \quad \text { for } n \in \mathbb{N} \text {. }
$$

It is clear that it is possible to choose a sequence of disks with these properties.

Next we choose a sequence $\left(r_{k}^{\prime}\right)$ satisfying $0<r_{k}^{\prime}<r_{k}$ for all $k \in \mathbb{N}$ such that with

$$
A^{\prime}=\mathbb{C} \backslash \bigcup_{j=1}^{\infty} D\left(a_{j}, r_{j}^{\prime}\right)
$$

we have

$$
\text { area }\left(A^{\prime} \cap P_{n}\right)<2
$$

for all $n \in \mathbb{N}$. For $k \in \mathbb{N}$ we put

$$
d_{k}=\min _{j \neq k} \operatorname{dist}\left(a_{k}, D\left(a_{j}, r_{j}\right)\right) .
$$

Note that $d_{k}>r_{k}$ for all $k \in \mathbb{N}$. We also choose a sequence $\left(\varepsilon_{k}\right)$ of positive real numbers such that

$$
\sum_{k=1}^{\infty} \varepsilon_{k}<\frac{1}{2}
$$

Finally we choose a sequence $\left(m_{k}\right)$ of positive integers such that

$$
\begin{gathered}
\frac{\varepsilon_{k} m_{k}}{r_{k}}>2, \\
\varepsilon_{k}\left(\frac{r_{k}}{r_{k}^{\prime}}\right)^{m_{k}}>3
\end{gathered}
$$

and

$$
\frac{m_{k}}{d_{k}}\left(\frac{r_{k}}{d_{k}}\right)^{m_{k}} \leq 1
$$

for all $k \in \mathbb{N}$ and

$$
\sum_{k \in I_{n}} \frac{r_{k}^{2}}{m_{k}} \leq \frac{3}{32}
$$


for all $n \in \mathbb{N}$. The function $f: \mathbb{C} \rightarrow \widehat{\mathbb{C}}$ is now defined by

$$
f(z)=\sum_{k=1}^{\infty} \varepsilon_{k}\left(\frac{r_{k}}{z-a_{k}}\right)^{m_{k}} .
$$

Lemma 8.1. The function $f$ is in $\mathcal{B}$ and $\infty$ is not an asymptotic value of $f$.

Proof. The derivative of $f$ is given by

$$
f^{\prime}(z)=-\sum_{k=1}^{\infty} \frac{\varepsilon_{k} m_{k}}{z-a_{k}}\left(\frac{r_{k}}{z-a_{k}}\right)^{m_{k}} .
$$

For $z \in D\left(a_{k}, r_{k}\right) \backslash\left\{a_{k}\right\}$ we thus have

$$
\left|f^{\prime}(z)\right| \geq \frac{\varepsilon_{k} m_{k}}{\left|z-a_{k}\right|}\left(\frac{r_{k}}{\left|z-a_{k}\right|}\right)^{m_{k}}-\sum_{\substack{j=0 \\ j \neq k}}^{\infty} \frac{\varepsilon_{j} m_{j}}{\left|z-a_{j}\right|}\left(\frac{r_{j}}{\left|z-a_{j}\right|}\right)^{m_{j}}
$$

and hence, using the definition of $d_{j}$,

$$
\left|f^{\prime}(z)\right| \geq \frac{\varepsilon_{k} m_{k}}{r_{k}}\left(\frac{r_{k}}{\left|z-a_{k}\right|}\right)^{m_{k}}-\sum_{\substack{j=0 \\ j \neq k}}^{\infty} \frac{\varepsilon_{j} m_{j}}{d_{j}}\left(\frac{r_{j}}{d_{j}}\right)^{m_{j}} .
$$

For $z \in D\left(a_{k}, r_{k}\right)$ we have $\left|z-a_{k}\right|<r_{k}$ and thus (8.3) yields

$$
\frac{\varepsilon_{k} m_{k}}{r_{k}}\left(\frac{r_{k}}{\left|z-a_{k}\right|}\right)^{m_{k}} \geq \frac{\varepsilon_{k} m_{k}}{r_{k}}>2 .
$$

On the other hand, applying (8.5) and (8.2) we obtain

$$
\sum_{\substack{j=0 \\ j \neq k}}^{\infty} \frac{\varepsilon_{j} m_{j}}{d_{j}}\left(\frac{r_{j}}{d_{j}}\right)^{m_{j}} \leq \sum_{j=1}^{\infty} \varepsilon_{j}<1 .
$$

It follows from (8.7), (8.8) and (8.9) that

$$
\left|f^{\prime}(z)\right| \geq \frac{1}{2} \frac{\varepsilon_{k} m_{k}}{r_{k}}\left(\frac{r_{k}}{\left|z-a_{k}\right|}\right)^{m_{k}}>1
$$

for all $z \in D\left(a_{k}, r_{k}\right) \backslash\left\{a_{k}\right\}$. The last inequality implies that all critical points of $f$ are contained in $A$. Since

$$
|f(z)| \leq \sum_{k=1}^{\infty} \varepsilon_{k}<\frac{1}{2} \quad \text { for } \quad z \in A,
$$

all critical and asymptotic values of $f$ are contained in $D\left(0, \frac{1}{2}\right)$. Hence $f \in \mathcal{B}$ and $\infty$ is not an asymptotic value of $f$.

We also note that $D(0,2) \subset A$ so that $f(D(0,2)) \subset D\left(0, \frac{1}{2}\right)$ by (8.11). Hence $D(0,2)$ contains an attracting fixed point and all singular values are contained in its basin of attraction.

Lemma 8.2. If $z \in \bigcup_{j=1}^{\infty} D\left(a_{j}, r_{j}^{\prime}\right)=\mathbb{C} \backslash A^{\prime}$, then $|f(z)|>2$. 
Proof. Let $z \in D\left(a_{k}, r_{k}^{\prime}\right)$. Proceeding as in the proof of Lemma 8.1, we obtain

$$
|f(z)| \geq \varepsilon_{k}\left(\frac{r_{k}}{\left|z-a_{k}\right|}\right)^{m_{k}}-\sum_{\substack{j=0 \\ j \neq k}}^{\infty} \varepsilon_{j}\left(\frac{r_{j}}{\left|z-a_{j}\right|}\right)^{m_{k}} \geq \varepsilon_{k}\left(\frac{r_{k}}{r_{k}^{\prime}}\right)^{m_{k}}-\sum_{j=0}^{\infty} \varepsilon_{j} .
$$

The conclusion now follows from (8.2) and (8.4).

Since sing $\left(f^{-1}\right) \subset D\left(0, \frac{1}{2}\right)$ we can define the branches of the inverse function of $f$ in every simply connected domain contained in $\mathbb{C} \backslash \overline{D(0,1)}$. Because of (8.11) such a branch of $f^{-1}$ maps this domain into $D\left(a_{k}, r_{k}\right)$ for some $k \in \mathbb{N}$.

Lemma 8.3. Let $g:\{w \in \mathbb{C}:|w|>1\} \backslash(-\infty,-1) \rightarrow D\left(a_{k}, r_{k}\right)$ be a branch of $f^{-1}$. Then

$$
\left|g^{\prime}(w)\right| \leq \frac{4 r_{k}}{m_{k}|w|}
$$

for $|w|>1$.

Proof. For $|w|>1$ we have

$$
\left|g^{\prime}(w)\right|=\frac{1}{\left|f^{\prime}(g(w))\right|} \leq \frac{2 r_{k}}{\varepsilon_{k} m_{k}}\left(\frac{\left|g(w)-a_{k}\right|}{r_{k}}\right)^{m_{k}}
$$

by (8.10). If $z \in D\left(a_{k}, r_{k}\right)$ and $|f(z)|>1$, then by (8.2),

$$
\begin{aligned}
|f(z)| & \leq \varepsilon_{k}\left(\frac{r_{k}}{\left|z-a_{k}\right|}\right)^{m_{k}}+\sum_{\substack{j=0 \\
j \neq k}}^{\infty} \varepsilon_{j}\left(\frac{r_{j}}{\left|z-a_{j}\right|}\right)^{m_{j}} \\
& \leq \varepsilon_{k}\left(\frac{r_{k}}{\left|z-a_{k}\right|}\right)^{m_{k}}+\sum_{\substack{j=0 \\
j \neq k}}^{\infty} \varepsilon_{j} \leq \varepsilon_{k}\left(\frac{r_{k}}{\left|z-a_{k}\right|}\right)^{m_{k}}+\frac{1}{2}|f(z)| .
\end{aligned}
$$

Thus

$$
|f(z)| \leq 2 \varepsilon_{k}\left(\frac{r_{k}}{\left|z-a_{k}\right|}\right)^{m_{k}}
$$

and with $w=f(z)$ we obtain

$$
|w| \leq 2 \varepsilon_{k}\left(\frac{r_{k}}{\left|g(w)-a_{k}\right|}\right)^{m_{k}} .
$$

It follows from (8.12) and (8.13) that

$$
\left|g^{\prime}(w)\right| \leq \frac{2 r_{k}}{\varepsilon_{k} m_{k}} \cdot \frac{2 \varepsilon_{k}}{|w|}=\frac{4 r_{k}}{m_{k}|w|} .
$$

We put $A^{*}=\{z \in A:|z|>2\}$.

Lemma 8.4. For $k, n \in \mathbb{N}$ we have

$$
\text { area }\left(f^{-k}\left(A^{*}\right) \cap P_{n}\right) \leq \frac{1}{2^{k}} .
$$


Proof. Let $g:\{w \in \mathbb{C}:|w|>1\} \backslash(-\infty,-1) \rightarrow D\left(a_{k}, r_{k}\right)$ be a branch of $f^{-1}$. By Lemma 8.3 we have

$$
\begin{aligned}
\iint_{A^{*}}\left|g^{\prime}(w)\right|^{2} d x d y & =\sum_{l=1}^{\infty} \iint_{A \cap P_{l}}\left|g^{\prime}(w)\right|^{2} d x d y \\
& \leq \sum_{l=1}^{\infty} \operatorname{area}\left(A \cap P_{l}\right) \cdot\left(\frac{4 r_{k}}{m_{k}}\right)^{2} \cdot \frac{1}{\left(2^{l}\right)^{2}} \\
& \leq \frac{16 r_{k}^{2}}{m_{k}^{2}} \sup _{l \geq 1} \operatorname{area}\left(A \cap P_{l}\right) \sum_{l=1}^{\infty} \frac{1}{2^{2 l}} \\
& =\frac{16}{3} \frac{r_{k}^{2}}{m_{k}^{2}} \sup _{l \geq 1} \operatorname{area}\left(A \cap P_{l}\right) .
\end{aligned}
$$

Noting that there are $m_{k}$ such branches of $f^{-1}$ we deduce from (8.6) that

$$
\text { area }\left(f^{-1}\left(A^{*}\right) \cap P_{n}\right) \leq \frac{16}{3} \sup _{l \geq 1} \text { area }\left(A \cap P_{l}\right) \sum_{k \in I_{n}} \frac{r_{k}^{2}}{m_{k}} \leq \frac{1}{2} \sup _{l \geq 1} \text { area }\left(A \cap P_{l}\right)
$$

for all $n \in \mathbb{N}$. Analogously we find that

$$
\text { area }\left(f^{-2}\left(A^{*}\right) \cap P_{n}\right) \leq \frac{1}{2} \sup _{l \geq 1} \text { area }\left(f^{-1}\left(A^{*}\right) \cap P_{l}\right)
$$

and induction yields that

$$
\text { area }\left(f^{-k}\left(A^{*}\right) \cap P_{n}\right) \leq \frac{1}{2^{k}} \sup _{l \geq 1} \operatorname{area}\left(A \cap P_{l}\right) \leq \frac{1}{2^{k}} .
$$

Put $B=\mathbb{C} \backslash \bigcup_{k=0}^{\infty} f^{-k}(A)$. Then

$$
\mathbb{C} \backslash B=\bigcup_{k=0}^{\infty} f^{-k}(A) \subset A^{\prime} \cup \bigcup_{k=0}^{\infty} f^{-k}\left(A^{*}\right) .
$$

Lemma 8.5. $\operatorname{area}(B)>0$.

Proof. It follows from Lemma 8.4 that

$$
\operatorname{area}\left(\bigcup_{k=1}^{\infty} f^{-k}\left(A^{*}\right) \cap P_{n}\right) \leq \sum_{k=0}^{\infty} \frac{1}{2^{k}}=2 .
$$

By (8.1) we have

$$
\text { area }\left(A^{\prime} \cap P_{n}\right)<2 \text {. }
$$

Thus (8.14) yields that area $\left(P_{n} \backslash B\right)<4$ and the conclusion follows.

Lemma 8.6. $\operatorname{area}(B \backslash I(f))=0$.

Proof. Suppose that area $(B \backslash I(f))>0$ and let $\xi$ be a density point of $B \backslash I(f)$. Since $\xi \in B$ we have $f^{m}(\xi) \in \mathbb{C} \backslash A$ and thus in particular $\left|f^{m}(\xi)\right|>2$ for $m \in \mathbb{N}$. As $\xi \notin I(f)$ there is a sequence $\left(m_{l}\right)$ tending to $\infty$ and a constant $R>0$ such that $\left|f^{m_{l}}(\xi)\right| \leq R$. Put $w_{l}=f^{m_{l}}(\xi)$. Passing to a subsequence if necessary we may assume that $w_{l} \rightarrow w$ where $2 \leq|w| \leq R$. Since the disks $D\left(a_{j}, r_{j}\right)$ have radius less than 1 we have

$$
\alpha:=\operatorname{area}(D(w, 1) \cap A)>0 .
$$


Since sing $\left(f^{-1}\right) \subset D\left(0, \frac{1}{2}\right)$ and since $f\left(D\left(0, \frac{1}{2}\right)\right) \subset f(A) \subset D\left(0, \frac{1}{2}\right)$ the branch $g_{l}$ of $f^{-m_{l}}$ which maps $w_{l}$ onto $\xi$ exists as a univalent function in $D\left(w, \frac{3}{2}\right)$. Fix $\delta$ with $0<\delta<\frac{1}{4}$ and choose $l$ so large that $\left|w_{l}-w\right| \leq \delta$. Put $D_{l}=D\left(w_{l}, 1+\delta\right)$. Then $D(w, 1) \subset D_{l} \subset D(w, 1+2 \delta)$ and Lemma 2.1 yields with $\lambda=2(1+2 \delta) / 3$ that

We obtain

$$
\sup _{u, v \in D_{l}} \frac{\left|g_{l}^{\prime}(u)\right|}{\left|g_{l}^{\prime}(v)\right|} \leq K:=\left(\frac{1+\lambda}{1-\lambda}\right)^{4}
$$

$$
\begin{aligned}
\operatorname{dens}\left(g_{l}(A), g_{l}\left(D_{l}\right)\right) & \geq \frac{1}{K^{2}} \operatorname{dens}\left(A, D_{l}\right) \\
& \geq \frac{1}{K^{2}} \operatorname{dens}\left(D(w, 1) \cap A, D_{l}\right)=\frac{\alpha}{K^{2} \pi(1+\delta)^{2}} .
\end{aligned}
$$

Lemma 2.1 also yields that there exist constants $\gamma_{1}, \gamma_{2}>0$ such that

$$
D\left(\xi, \gamma_{1}\left|g_{l}^{\prime}\left(w_{l}\right)\right|\right) \subset g_{l}\left(D_{l}\right) \subset D\left(\xi, \gamma_{2}\left|g_{l}^{\prime}\left(w_{l}\right)\right|\right) .
$$

With $r_{l}=\gamma_{2}\left|g_{l}^{\prime}\left(w_{l}\right)\right|$ it follows that

$$
\operatorname{dens}\left(g_{l}(A), D\left(\xi, r_{l}\right)\right)>\frac{\gamma_{1}^{2} \alpha}{\gamma_{2}^{2} K^{2} \pi(1+\delta)^{2}} .
$$

Also, (8.10) shows that $g_{m_{l}}^{\prime}\left(w_{l}\right) \rightarrow 0$ and hence $r_{l} \rightarrow 0$ as $l \rightarrow \infty$. Since $g_{l}(A) \cap B=\emptyset$ for all $l \in \mathbb{N}$ this contradicts the assumption that $\xi$ is a density point of $B$.

Theorem 1.4 follows from Lemmas 8.5 and 8.6 .

\section{ACKNowledgement}

We thank the referees for a great number of valuable suggestions.

\section{REFERENCES}

[1] S. B. Bank and R. P. Kaufman, On meromorphic solutions of first-order differential equations. Comment. Math. Helv. 51 (1976), 289-299. MR0430370 (55:3375)

[2] K. Barański, Hausdorff dimension of hairs and ends for entire maps of finite order. Math. Proc. Cambridge Philos. Soc. 145 (2008), 719-737. MR2464786 (2009m:37127)

[3] K. Barański, B. Karpińska and A. Zdunik, Hyperbolic dimension of Julia sets of meromorphic maps with logarithmic tracts. Int. Math. Res. Not. IMRN 2009, 615-624. MR2480096 (2009k:37109)

[4] W. Bergweiler, Iteration of meromorphic functions. Bull. Amer. Math. Soc. (N. S.) 29 (1993), 151-188. MR1216719 (94c:30033)

[5] W. Bergweiler, P. J. Rippon and G. M. Stallard, Dynamics of meromorphic functions with direct or logarithmic singularities. Proc. London Math. Soc. 97 (2008), 368-400. MR2439666 (2010b:37122)

[6] P. Domínguez, Dynamics of transcendental meromorphic functions. Ann. Acad. Sci. Fenn. Math. 23 (1998), 225-250. MR1601879 (99b:30031)

[7] A. E. Eremenko, On the iteration of entire functions, in "Dynamical systems and ergodic theory". Banach Center Publications 23, Polish Scientific Publishers, Warsaw 1989, pp. 339345. MR1102727 (92c:30027)

[8] A. Eremenko, Transcendental meromorphic functions with three singular values. Illinois J. Math. 48 (2004), 701-709. MR2085435 (2005f:30056)

[9] A. E. Eremenko and M. Yu. Lyubich, Dynamical properties of some classes of entire functions. Ann. Inst. Fourier 42 (1992), 989-1020. MR1196102 (93k:30034)

[10] A. A. Goldberg and I. V. Ostrovskii, Value distribution of meromorphic functions. Transl. Math. Monographs 236, American Math. Soc., Providence, RI, 2008. MR2435270 (2009f:30067) 
[11] W. K. Hayman, Meromorphic functions. Clarendon Press, Oxford, 1964. MR0164038 (29:1337)

[12] J. Kotus, On the Hausdorff dimension of Julia sets of meromorphic functions, II. Bull. Soc. Math. France 123 (1995), 33-46. MR 1330786 (96i:30017)

[13] J. Kotus and M. Urbański, Hausdorff dimension and Hausdorff measures of Julia sets of elliptic functions. Bull. London Math. Soc. 35 (2003), 269-275. MR1952406 (2003j:37067)

[14] J. Kotus and M. Urbański, Fractal measures and ergodic theory of transcendental meromorphic functions, in "Transcendental Dynamics and Complex Analysis". London Math. Soc. Lect. Note Ser. 348. Edited by P. J. Rippon and G. M. Stallard, Cambridge Univ. Press, Cambridge, 2008, pp. 251-316. MR2458807(2010h:37099)

[15] J. K. Langley, Critical values of slowly growing meromorphic functions. Comput. Methods Funct. Theory 2 (2002), 537-547. MR2038137(2005a:30055)

[16] D. Mauldin and M. Urbański, Dimensions and measures in infinite iterated function systems. Proc. London Math. Soc. (3) 73 (1996), 105-154. MR1387085 (97c:28020)

[17] V. Mayer, The size of the Julia set of meromorphic functions. Math. Nachr. 282 (2009), 1189-1194. MR 2547715

[18] C. McMullen, Area and Hausdorff dimension of Julia sets of entire functions. Trans. Amer. Math. Soc. 300 (1987), 329-342. MR871679(88a:30057)

[19] R. Nevanlinna, Eindeutige analytische Funktionen. Springer, Berlin, Heidelberg, 1953. MR0057330 (15:208c)

[20] L. Rempe and S. van Strien, Absence of line fields and Mañé's theorem for nonrecurrent transcendental functions. Trans. Amer. Math. Soc. 363 (2011), 203-228. MR.2719679

[21] P. J. Rippon and G. M. Stallard, Iteration of a class of hyperbolic meromorphic functions. Proc. Amer. Math. Soc. 127 (1999), 3251-3258. MR1610785 (2000b:30033)

[22] H. Schubert, Über die Hausdorff-Dimension der Juliamenge von Funktionen endlicher Ordnung. Dissertation, University of Kiel, 2007.

[23] G. M. Stallard, Entire functions with Julia sets of zero measure. Math. Proc. Cambridge Philos. Soc. 108 (1990), 551-557. MR1068456 (91g:30037)

[24] G. M. Stallard, The Hausdorff dimension of Julia sets of meromorphic functions. J. London Math. Soc. (2) 49 (1994), 281-295. MR1260113 (95b:58127)

[25] G. M. Stallard, Dimensions of Julia sets of transcendental meromorphic functions, in "Transcendental Dynamics and Complex Analysis". London Math. Soc. Lect. Note Ser. 348. Edited by P. J. Rippon and G. M. Stallard, Cambridge Univ. Press, Cambridge, 2008, pp. 425-446. MR2458811 (2010h:37101)

[26] O. Teichmüller, Eine Umkehrung des zweiten Hauptsatzes der Wertverteilungstheorie. Deutsche Math. 2 (1937), 96-107; Gesammelte Abhandlungen, Springer, Berlin, Heidelberg, New York, 1982, pp. 158-169.

Mathematisches Seminar, Christian-Albrechts-Universität Zu Kiel, Ludewig-MeynStr. 4, D-24098 Kiel, Germany

E-mail address: bergweiler@math.uni-kiel.de

Faculty of Mathematics and Information Science, Warsaw University of Technology, Pl. Politechniki 1, 00-661 Warszawa, Poland

E-mail address: J.Kotus@impan.pl 